An overview of the design, construction and performance of large area triple-GEM prototypes for future upgrades of the CMS forward muon system

This article has been downloaded from IOPscience. Please scroll down to see the full text article.

2012 JINST 7 C05008

(http://iopscience.iop.org/1748-0221/7/05/C05008)

View the table of contents for this issue, or go to the journal homepage for more

Download details:

IP Address: 157.193.98.16

The article was downloaded on 20/06/2013 at 09:04

Please note that terms and conditions apply. 
$2^{\text {nd }}$ International Conference on Micro Pattern Gaseous Detectors, 29 AUGUST - 1 SEPTEMBER 2011,

KOBE, JAPAN

\section{An overview of the design, construction and performance of large area triple-GEM prototypes for future upgrades of the CMS forward muon system}

D. Abbaneo, ${ }^{a}$ M. Abbrescia, ${ }^{b}$ M. Alfonsi, ${ }^{a}$ C. Armaingaud, ${ }^{a}$ P. Aspell, ${ }^{a}$ M.G. Bagliesi,${ }^{i}$ Y. Ban, ${ }^{c}$ S. Bally, ${ }^{a}$ L. Benussi, ${ }^{f}$ U. Berzano, ${ }^{a}$ S. Bianco, ${ }^{f}$ J. Bos,${ }^{a}$ K. Bunkowski, ${ }^{a}$ J. Cai, ${ }^{c}$ R. Cecchi, ${ }^{i}$ J.P. Chatelain, ${ }^{a}$ J. Christiansen, ${ }^{a}$ S. Colafranceschi, ${ }^{f}$

A. Colaleo, ${ }^{b}$ A. Conde Garcia, ${ }^{a}$ G. Croci, ${ }^{a}$ E. David, ${ }^{a}$ G. de Robertis, ${ }^{b}$

R. De Oliveira, ${ }^{a}$ S. Dildick, ${ }^{g}$ S. Duarte Pinto, ${ }^{a}$ S. Ferry, ${ }^{a}$ F. Formenti, ${ }^{a}$ L. Franconi, ${ }^{a}$

T. Fruboes, ${ }^{k}$ K. Gnanvo, ${ }^{h}$ A. Gutierrez, ${ }^{e}$ M. Hohlmann, ${ }^{h}$ P.E. Karchin, ${ }^{e}$ F. Loddo, ${ }^{b}$

G. Magazzù, ${ }^{i}$ M. Maggi, ${ }^{b}$ A. Marchioro, ${ }^{a}$ A. Marinov, ${ }^{g}$ K. Mehta, ${ }^{a}$ J. Merlin, ${ }^{a}$

A. Mohapatra, ${ }^{d}$ T. Moulik, ${ }^{d}$ M.V. Nemallapudi, ${ }^{a}$ S. Nuzzo,${ }^{b}$ E. Oliveri,${ }^{i}$ D. Piccolo, ${ }^{f}$

S. Park, ${ }^{l}$ H. Postema, ${ }^{a}$ G. Raffone, ${ }^{f}$ A. Rodrigues, ${ }^{a}$ L. Ropelewski, ${ }^{a}$ G. Saviano, ${ }^{f}$

A. Sharma, ${ }^{a}, 1$ W. Snoeys, ${ }^{a}$ M.J. Staib, ${ }^{h}$ M. van Stenis, ${ }^{a}$ H. Teng, ${ }^{c}$ M. Tytgat, ${ }^{g}$

S.A. Tupputi, ${ }^{b}$ N. Turini, ${ }^{i}$ N. Smilkjovic, ${ }^{a}$ M. Villa, ${ }^{a}$ N. Zaganidis ${ }^{g}$ and M. Zientek ${ }^{a}$

${ }^{a}$ CERN, Geneva, Switzerland

${ }^{b}$ Politecnico di Bari, Università di Bari and INFN Sezione di Bari, Bari, Italy

${ }^{c}$ Peking University, Beijing, China

${ }^{d}$ NISER, Bhubaneswar, India

${ }^{e}$ Wayne State University, Detroit, U.S.A.

${ }^{f}$ Laboratori Nazionali di Frascati INFN, Frascati, Italy

${ }^{g}$ Department of Physics and Astronomy, Universiteit Gent, Gent, Belgium

${ }^{h}$ Florida Institute of Technology, Melbourne, U.S.A.

${ }^{i}$ INFN Sezione di Pisa, Pisa, Italy

${ }^{j}$ University of Wisconsin, Madison, U.S.A.

${ }^{k}$ Narodowe Centrum Badan Jadrowych (NCBJ), Otwock, Świerk, Poland

${ }^{l}$ KODEL, Korea University, Seoul, South Korea

E-mail: archana.sharma@cern.ch

\footnotetext{
${ }^{1}$ Corresponding author.
} 
ABSTRACT: GEM detectors are used in high energy physics experiments given their good spatial resolution, high rate capability and radiation hardness. An international collaboration is investigating the possibility of covering the $1.6<|\eta|<2.4$ region of the CMS muon endcaps with large-area triple-GEM detectors. The CMS high- $\eta$ area is actually not fully instrumented, only Cathode Strip Chamber (CSC) are installed. The vacant area presents an opportunity for a detector technology able to to cope with the harsh radiation environment; these micropattern gas detectors are an appealing option to simultaneously enhance muon tracking and triggering capabilities in a future upgrade of the CMS detector. A general overview of this feasibility study is presented. Design and construction of small $(10 \mathrm{~cm} \times 10 \mathrm{~cm})$ and full-size trapezoidal $(1 \mathrm{~m} \times 0.5 \mathrm{~m})$ triple-GEM prototypes is described. Results from measurements with $x$-rays and from test beam campaigns at the CERN SPS is shown for the small and large prototypes. Preliminary simulation studies on the expected muon reconstruction and trigger performances of this proposed upgraded muon system are reported.

KEYWORDS: Muon spectrometers; Trigger concepts and systems (hardware and software); Trigger detectors 


\section{Contents}

1 Introduction 1

2 High momentum muon trigger and tracking resolution improvements with tripleGEMs for forward high eta muons

3 Construction and performance of small and full scale prototypes 4

4 Prospects for industrial production 9

5 Assembly techniques 9

6 Conclusion and outlook 9

\section{Introduction}

After the first few years of LHC running, the operation of the CMS [2] detector will set a challenging scenario in which about $300 \mathrm{fb}^{-1}$ of collision data will have been collected already, and the machine will operate at a centre-of-mass energy of $14 \mathrm{TeV}$, with a luminosity of of $2-3 \times 10^{34} \mathrm{~cm}^{-2} \mathrm{~s}^{-1}$ and a nominal or twice the nominal amount of pile-up. The forward muon system needs to operate efficiently, triggering on and identifying muons in order to study e.g. boosted objects and forward-backward asymmetries, coupling \& spin measurements and fusion processes.

The muon system of CMS comprises five barrel wheels and four endcap muon stations with a coverage up to $\eta=1.6$ (2.4 considering only CSCs) in the forward region. The GEM Collaboration (GEMs for CMS) [1, 3-5] proposes augmenting the $1.6<\eta<2.4$ endcap region with muon triggering and tracking, showcasing the triple-GEM as a strongly favoured option for enhanced tracking in a high density region, extending the CMS tracking reach to the forward zone. These micropattern detectors have matured over the last decade and have been exploited by various experiments in a harsh radiation environment with high particle rates not only for tracking but also for trigger generation [6].

Examining this table we can see that triple-GEMs in CMS would be very robust in terms of rate capability and radiation hardness, due to the experience and measurements demonstrated in LHCb measurements [7], with these numbers being $\approx \mathrm{MHz} / \mathrm{mm}^{2}$ and $20 \mathrm{C} / \mathrm{cm}$ respectively. Table 1 illustrates the detector performance requirements for various eta sectors, and shows that triple-GEMs in CMS, with measured values in LHCb [7] of $\approx \mathrm{MHz} / \mathrm{mm}^{2}$ and $20 \mathrm{C} / \mathrm{cm}^{2}$, would be very robust in terms of rate capability and radiation hardness. 


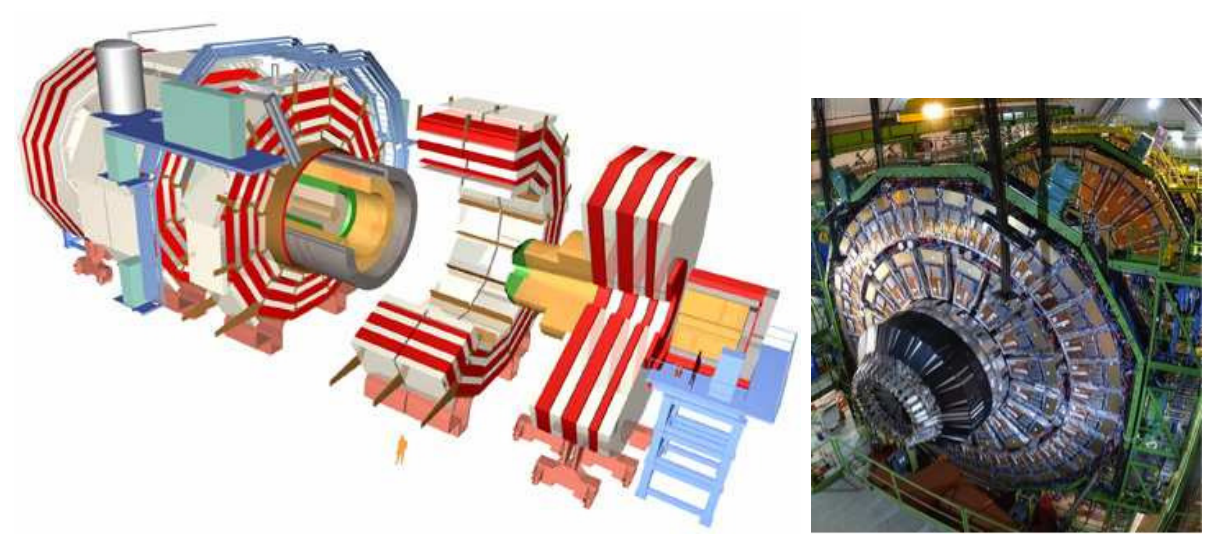

Figure 1. The CMS Experiment.

Table 1. Performance requirements in the various $\eta$ sectors.

\begin{tabular}{|c|c|c|c|}
\hline Region & $\begin{array}{c}\text { Rates }\left(\mathrm{Hz} / \mathrm{cm}^{2}\right) \\
\text { LHC }\left(10^{34} \mathrm{~cm}^{2} / \mathrm{s}\right)\end{array}$ & $\begin{array}{l}\text { High Luminosity LHC } \\
2-3 \times \text { LHC }\end{array}$ & $\begin{array}{c}\text { Phase II } \\
\left(10^{35} \mathrm{~cm}^{2} / \mathrm{s}\right)\end{array}$ \\
\hline Barrel RPC & 30 & Few 100 & $\approx \mathrm{kHz}(\mathrm{tbc})$ \\
\hline Endcap RPC 1, 2, 3,4 $\eta<1.6$ & 30 & Few 100 & $\approx \mathrm{kHz}(\mathrm{tbc})$ \\
\hline Expected Charge in 10 years & $0.05 \mathrm{C} / \mathrm{cm}^{2}$ & $0.15 \mathrm{C} / \mathrm{cm}^{2}$ & $\approx \mathrm{C} / \mathrm{cm}^{2}$ \\
\hline Endcap RPC $1,2,3,4 \eta>1.6$ & $500 \mathrm{~Hz} \approx \mathrm{kHz}$ & few $\mathrm{kHz}$ & few $10 \mathrm{~s} \mathrm{kHz}$ \\
\hline Total Expected Charge in 10 years & $0.05-1 \mathrm{C} / \mathrm{cm}^{2}$ & few $\mathrm{C} / \mathrm{cm}^{2}$ & few $10 \mathrm{~s} \mathrm{C} / \mathrm{cm}^{2}$ \\
\hline
\end{tabular}

\section{High momentum muon trigger and tracking resolution improvements with triple- GEMs for forward high eta muons}

Simulations prove that a CMS muon system upgrade with triple-GEMs would bring significant improvements to its triggering and tracking capabilities [8]. Tests with triple-GEM prototypes have demonstrated a time resolution commensurate with the requirements listed in table 1 . The expected spatial resolution of triple-GEMs reaches a turning point from a uniform to Gaussian behavior (figure 2), for reconstruction of high momenta muons. With an improved high granularity, muon momentum resolution would also improve as shown in figure 2 .

The trigger system in CMS [9] is based on robust muon identification and reconstruction at a record magnetic field of $3.8 \mathrm{~T}$ [2] with an iron yoke. For the forward muon system however, the magnetic field return causes ambiguity in $\Delta \Phi$ and a fourfold ambiguity as we cross the first two disks [10]. Hence, more information is needed to disentangle and improve the pattern recognition with a high $\mathrm{R} \Delta \phi$ granularity. The high $\eta$ trigger performance strongly relies on spatial information and system coverage redundancy, and loss of chambers in key regions can be detrimental. In addition the missing chambers contribute towards the inefficiency and high rate particle of fake triggers. In order to address these issues, and improve tracking and triggering (figure 3) in CMS Muon system, we have undertaken the study of triple-GEMs. 

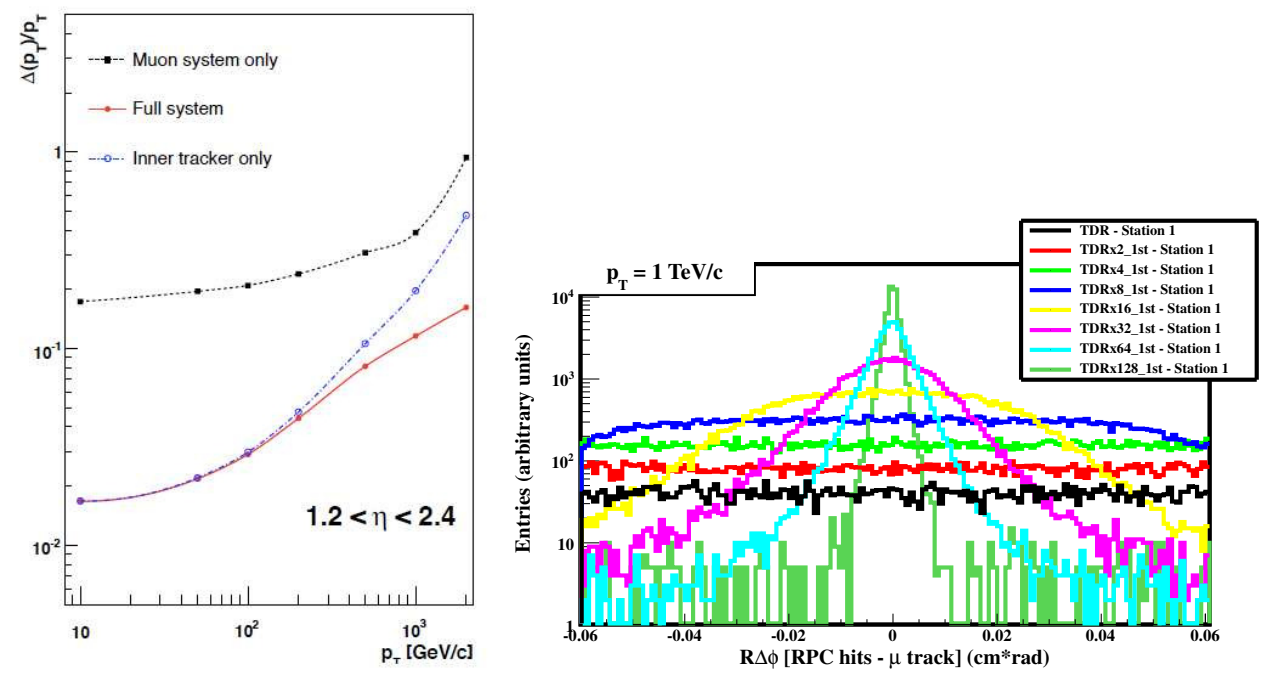

Figure 2. Momentum resolution (left) and spatial residuals distributions comparison for $\mathrm{p}_{\mathrm{T}}=1 \mathrm{TeV} / \mathrm{c}$ muons.
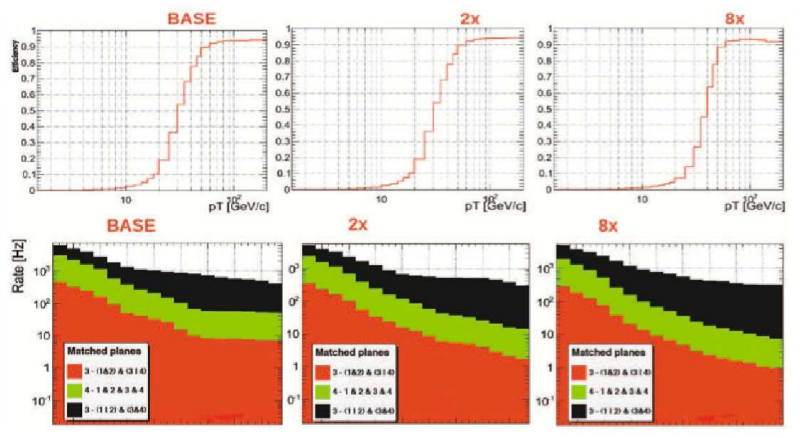

$8 \times$


Figure 3. Top: Trigger turn-on curves for $\mathrm{pT}_{i} 50 \mathrm{GeV} / \mathrm{c}$ threshold. Bottom: Trigger rates vs $\mathrm{p}_{\mathrm{T}}$ thresholds.; (right) Momentum resolution vs spatial resolution for different muon momenta.

In figure 4 we can see the components of a triple-GEM with (a) the copper clad Kapton GEM foil, in (b) the field configuraton of the electron multiplier with appropriate voltage applied to the top and bottom electrodes. Arranging three GEMs in tandem as shown in figure 4(c), yields a tripleGEM detector, Fig 4(d) demonstrates that the this type of detector can operate at a gain up to $\approx 10^{4}$. 

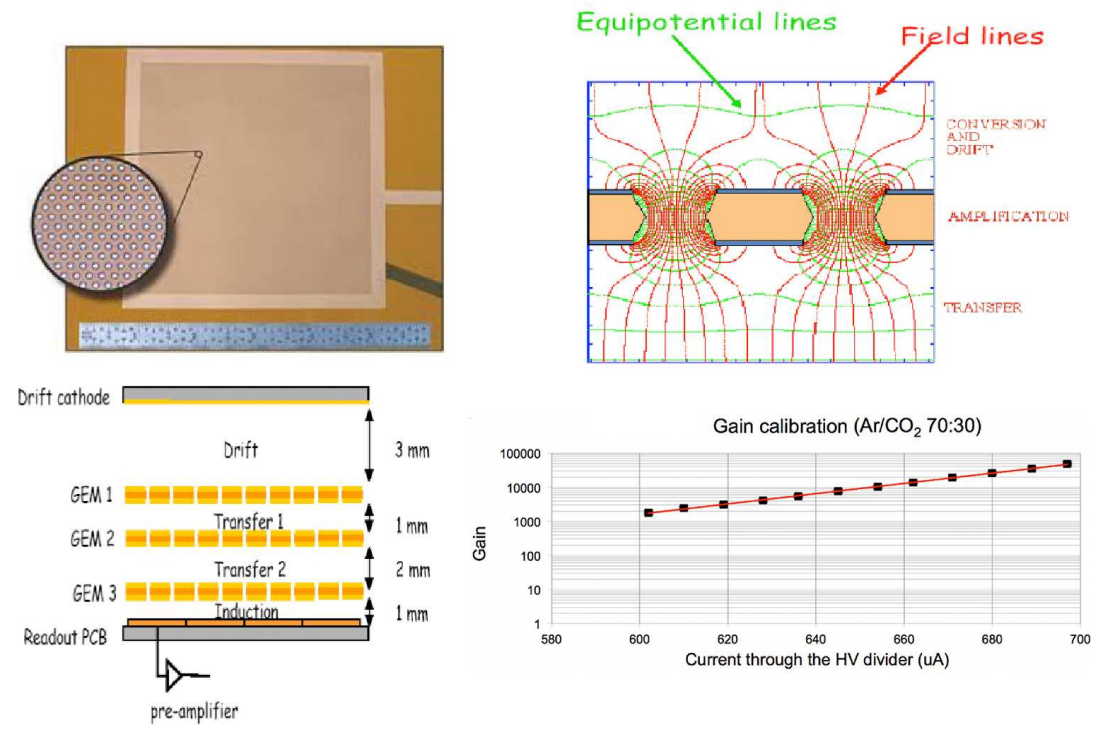

Figure 4. Components and operation of a triple-GEM.

With a micropattern detector like the triple-GEM it is feasible to combine triggering and tracking functions. The readout may be tailored and optimized to enhance the $(\eta-\phi)$ granularity exploiting the rate capability $\approx 10^{5} / \mathrm{mm}^{2}$ and spatial resolution in the order of $100 \mu \mathrm{m}$. With a time resolution of $\approx 4-5$ ns operating at full efficiency with a non-flammable gas mixture, this detector technology promises to fulfill all requirements including operation at a high magnetic field. In the following we demonstrate the operation and study of small and large scale prototypes and corroborate the requirements for a high eta tracking and trigger complement for the CMS forward muon detector.

\section{Construction and performance of small and full scale prototypes}

In the last two years several prototypes have been built and extensively studied [3, 4]. Here some results will be reported. In table 2 and 3, we show the list of prototypes that were constructed and studied, including the detector configuration.

Typically, GEM foils have been made using standard double mask technology [11], with the limitation that going from small to large sizes, the two masks need to be carefully aligned, which limits the production of large sizes. Single mask GEM foil technology has been successfully implemented, and GEM detectors made with this technology have been demonstrated to work equally well [13]. In figure 5 we see beam test results from standard GEMs and single mask GEMs with various operational gas mixtures with almost full efficiencies and a wide efficiency plateau [5]. We took this technology a step further and built large size detectors commensurate with requirements in CMS in the first muon station, the dimensions being about $1 \mathrm{~m} \times 0.5 \mathrm{~m}$. The readout strips are also trapezoidal like the detector shown in figure 6 . They are narrow $(\approx 0.6 \mathrm{~mm})$ at the high- $\eta$ end and wider $(\approx 1.2 \mathrm{~mm})$ at the low eta end of the detector. The detector configuration is shown in figure 7 . 


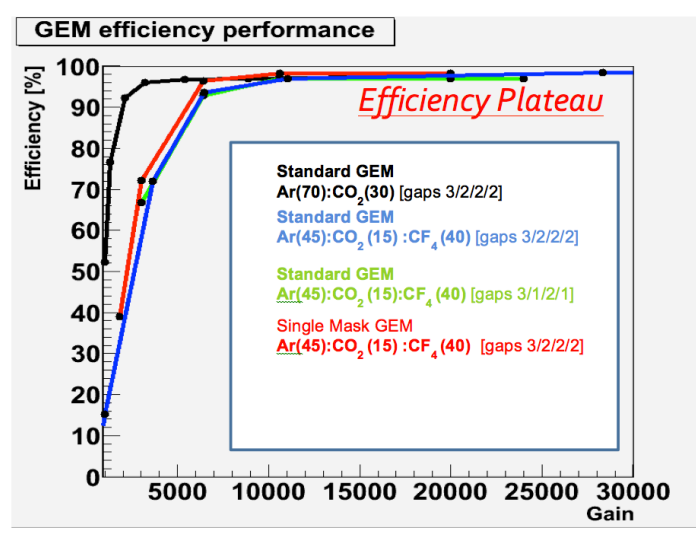

Figure 5. Performance of $10 \times 10$ GEMs made with standard and single mask techniques.


Figure 6. Left: Gain of triple-GEM GE11-1 full size prototype. Right: GE1/1_I installed at H4 beam line at the Super Proton Syncrotron (SPS).

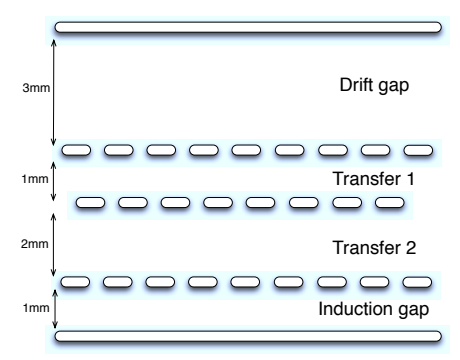

Figure 7. GE11-2 gap configuration.

This prototype was tested in the laboratory and a gain was measured as shown in figure 6(right) for two different sectors. Furthermore, this detector was placed in a magnetic field at the M1 magnet at the CERN SPS beam area as shown in figure 8(right), the displacement due to the magnetic field as a function of magnetic field is shown in figure 8(left). The efficiency, space resolution and cluster size are shown in figure 9, 10. The readout electronics used for these measurements is based on the VFAT2 chip [12], originally developed for the TOTEM experiment [6]. 

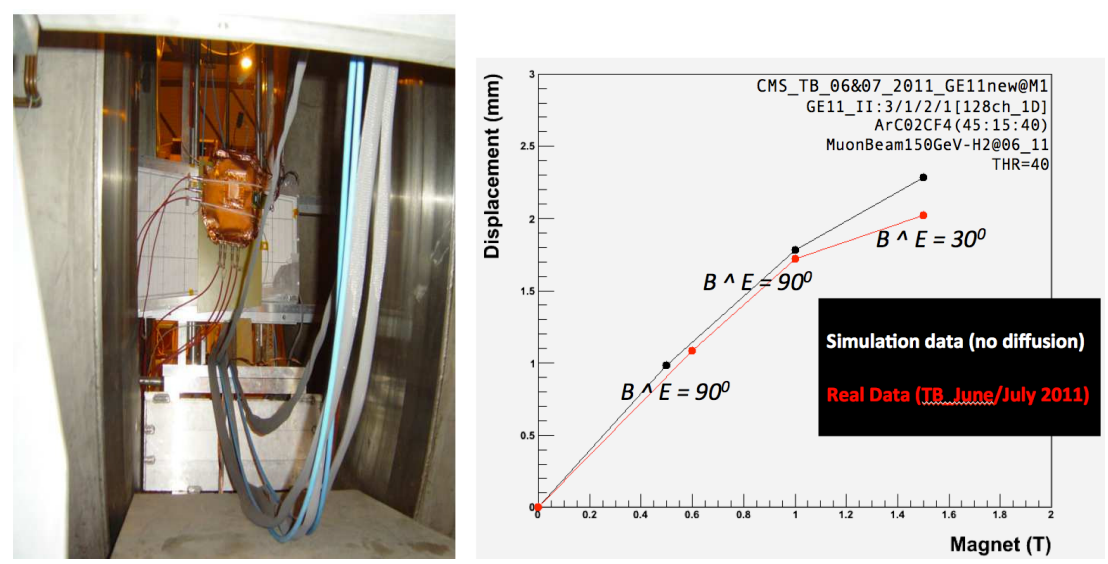

Figure 8. Left: GE1/1_II performance inside a strong magnetic field: strip cluster displacement due to the magnetic field. Right: GE1/1_II installed inside the M1 magnet at the H2 beam line at the SPS.
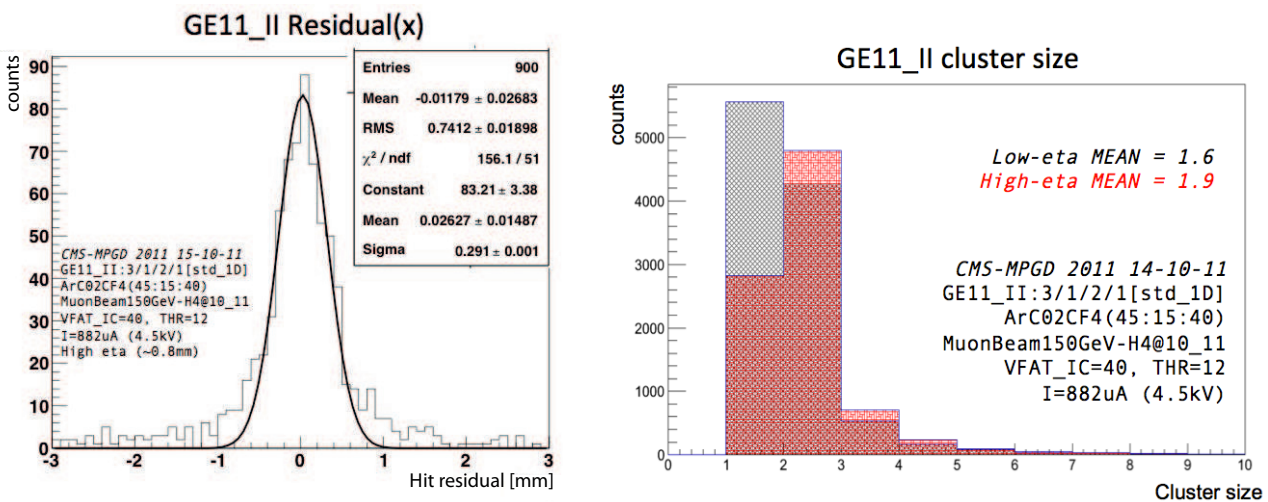

Figure 9. GE1/1_II performance: space resolution and cluster size in the high and low $\eta$.
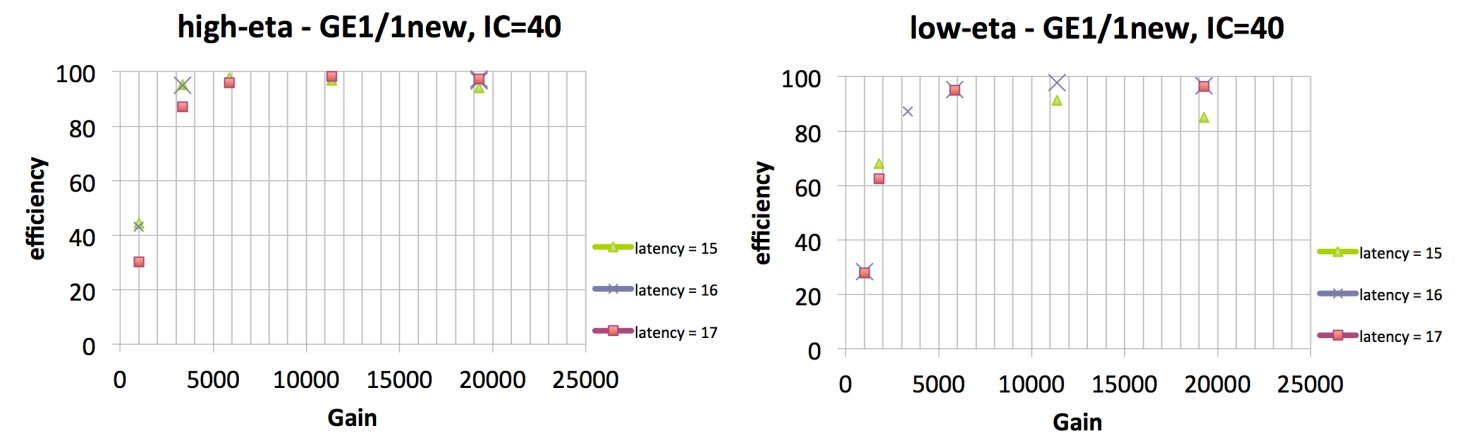

Figure 10. GE1/1_II efficiency performance at high and low $\eta$ ends. Efficiency reaches $96.5 \%$ at gain 7000. 
Table 2. Overview of all the prototypes that were constructed and studied in this project.

\begin{tabular}{|c|c|c|c|c|c|c|c|c|}
\hline \multirow{9}{*}{$\sqrt{2}$} & Name & $\begin{array}{l}\text { Mask } \\
\text { Type }\end{array}$ & $\begin{array}{l}\text { Prod. } \\
\text { Tech. }\end{array}$ & $\begin{array}{l}\text { Active } \\
\text { Area } \\
\left(\mathrm{mm}^{2}\right)\end{array}$ & $\begin{array}{c}\text { Readout/pitch } \\
\text { number of dimensions/mm }\end{array}$ & $\begin{array}{c}\text { Gap Sizes } \\
(\text { drift, tr. 1, tr. 2, ind.) } \\
(\mathrm{mm} / \mathrm{mm} / \mathrm{mm} / \mathrm{mm})\end{array}$ & \#strips & $\begin{array}{l}\text { Prod. } \\
\text { Site }\end{array}$ \\
\hline & Timing GEM & double-mask & standard & $100 \times 100$ & 1D 0.8 & $3 / 2 / 2 / 2 \& 3 / 1 / 2 / 1$ & 128 & CERN \\
\hline & Single-Mask GEM & single-mask & standard & $100 \times 100$ & 2D 0.4 & $3 / 2 / 2 / 2$ & 512 & CERN \\
\hline & Honeycomb GEM & double-mask & standard & $100 \times 100$ & 2D 0.4 & $3 / 2 / 2 / 2$ & 512 & CERN \\
\hline & CMS Proto I & single-mask & self-stretching & $990 \times(220-450)$ & 1D 0.8-1.6 & $3 / 2 / 2 / 2$ & 1024 & CERN \\
\hline & CMS Proto II & single-mask & self-stretching & $990 \times(220-450)$ & 1D $0.6-1.2$ & $3 / 1 / 2 / 1$ & 3184 & CERN \\
\hline & CMS Proto III & single-mask & self-stretching & $100 \times 100$ & 1D 0.4 & $3 / 1 / 2 / 1$ & 256 & CERN \\
\hline & CMS Proto IV & single-mask & self-stretching & $300 \times 300$ & 1D $0.6-1.8$ & $3 / 1 / 2 / 1$ & 256 & CERN \\
\hline & Korean I & double-mask & standard & $80 \times 80$ & 1D 0.6 & $3 / 1 / 2 / 1$ & 256 & New Flex [14] \\
\hline
\end{tabular}


Table 3. Overview of all the prototypes performance.

\begin{tabular}{|c|c|c|c|c|c|c|c|}
\hline Name & Max Gain & Gas Mix. & Electronics & $\begin{array}{c}\text { Efficiency } \\
(\%)\end{array}$ & $\begin{array}{l}\text { Space resolution } \\
(\mathrm{mm})\end{array}$ & $\begin{array}{c}\text { Time resolution } \\
(\mathrm{ns})\end{array}$ & $\begin{array}{l}\text { Magnet } \\
\qquad(\mathrm{T})\end{array}$ \\
\hline \multirow[t]{2}{*}{ Timing GEM } & 60776 & $\mathrm{Ar} / \mathrm{CO}_{2}(70: 30)$ & VFAT & 98.3 & 0.24 & 9.8 & $\mathrm{~B}=0$ \\
\hline & 17943 & $\mathrm{Ar} / \mathrm{CO}_{2} / \mathrm{CF}_{4}(45: 15: 40)$ & VFAT & 98.8 & 0.24 & 4.5 & $\mathrm{~B}=0$ \\
\hline \multirow[t]{2}{*}{ Single-Mask GEM } & & $\mathrm{Ar} / \mathrm{CO}_{2}(70: 30)$ & VFAT & 97.9 & & & $\mathrm{~B}=0$ \\
\hline & & $\mathrm{Ar} / \mathrm{CO}_{2} / \mathrm{CF}_{4}(45: 15: 40)$ & VFAT & 98.6 & & & $\mathrm{~B}=0$ \\
\hline \multirow[t]{2}{*}{ Honeycomb GEM } & & $\mathrm{Ar} / \mathrm{CO}_{2}(70: 30)$ & VFAT & & & & $\mathrm{B}=0$ \\
\hline & & $\mathrm{Ar} / \mathrm{CO}_{2} / \mathrm{CF}_{4}(45: 15: 40)$ & VFAT & 70.5 & & & $\mathrm{~B}=0$ \\
\hline \multirow[t]{2}{*}{ CMS Proto I } & 15889 & $\mathrm{Ar} / \mathrm{CO}_{2}(70: 30)$ & VFAT & 98.8 & & & $\mathrm{~B}=0$ \\
\hline & & $\mathrm{Ar} / \mathrm{CO}_{2} / \mathrm{CF}_{4}(45: 15: 40)$ & VFAT & 99.0 & 0.32 & & $\mathrm{~B}=0$ \\
\hline \multirow[t]{2}{*}{ CMS Proto II } & 15889 & $\mathrm{Ar} / \mathrm{CO}_{2}(70: 30)$ & VFAT\&APV & 98.9 & & & $\mathrm{~B}=1.5$ \\
\hline & 18938 & $\mathrm{Ar} / \mathrm{CO}_{2} / \mathrm{CF}_{4}(45: 15: 40)$ & VFAT\&APV & 97.8 & 0.29 & & $\mathrm{~B}=1.5$ \\
\hline \multirow[t]{2}{*}{ CMS Proto III } & & $\mathrm{Ar} / \mathrm{CO}_{2}(70: 30)$ & VFAT & & & & $\mathrm{B}=0$ \\
\hline & & $\mathrm{Ar} / \mathrm{CO}_{2} / \mathrm{CF}_{4}(45: 15: 40)$ & VFAT & & & & $\mathrm{B}=0$ \\
\hline \multirow[t]{2}{*}{ CMS Proto IV } & & $\mathrm{Ar} / \mathrm{CO}_{2}(70: 30)$ & VFAT & & & & $\mathrm{B}=0$ \\
\hline & & $\mathrm{Ar} / \mathrm{CO}_{2} / \mathrm{CF}_{4}(45: 15: 40)$ & VFAT & & & & $\mathrm{B}=0$ \\
\hline \multirow[t]{2}{*}{ Korean I } & 4653 & $\mathrm{Ar} / \mathrm{CO}_{2}(70: 30)$ & VFAT & & & & $\mathrm{B}=0$ \\
\hline & & $\mathrm{Ar} / \mathrm{CO}_{2} / \mathrm{CF}_{4}(45: 15: 40)$ & VFAT & & & & $\mathrm{B}=0$ \\
\hline
\end{tabular}



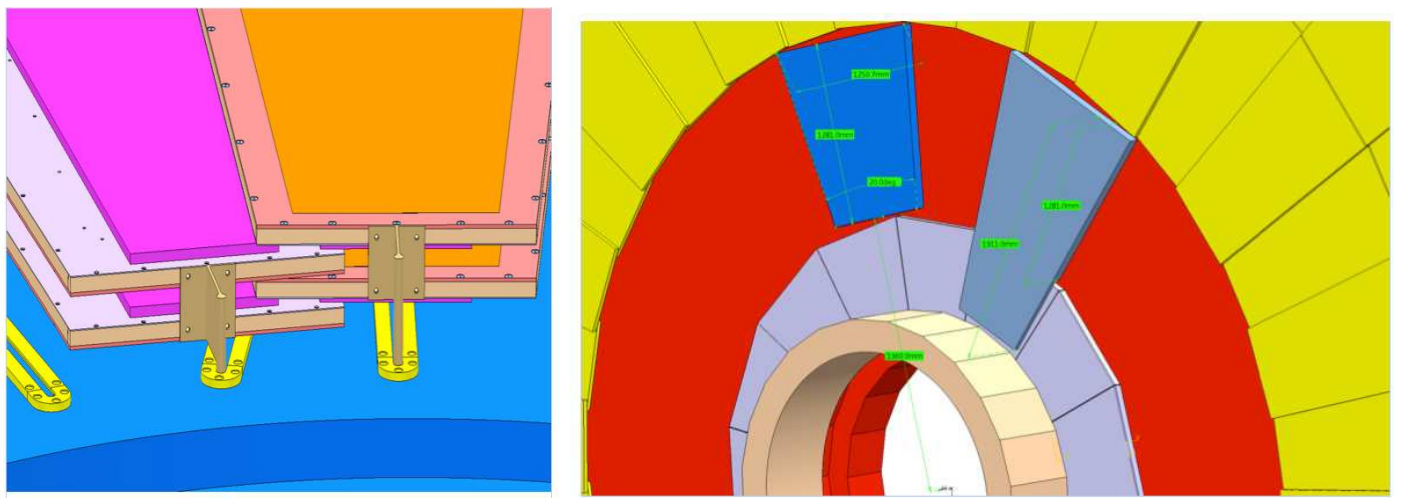

Figure 11. Envelope studies for station 1 and 2 in CMS high eta muon system; detector in muon station 1 are $\approx 1 \mathrm{~m} \times 0.5 \mathrm{~m}$, while in the second muon station has been located and small GEMs have already been produced.

\section{Prospects for industrial production}

Instrumenting large muon stations (the first and second endcap disks) of the CMS forward muons with triple-GEMs will require several $100 \mathrm{~m}^{2}$ of GEM foils. In figure 11 we can see the large envelope in CMS that will need to be instrumented.

Presently GEM foils are fabricated in the printed circuit workshop at CERN. In view of the large production volume, it is important to also look for industrial partners. GEM foils produced by a Korean company have been tested and showed encouraging results in terms of fabrication quality and gain performance (figure 12).

\section{Assembly techniques}

The cost of the whole detector station will be driven by construction and assembly expenses. We have investigated new assembly techniques for triple GEMs which do not need any glue, as this would reduce the assembly time from a few days to a few hours. Figure 13 shows some pictures of the assembly of a $10 \times 10 \mathrm{~cm}^{2}$ triple-GEM detector using the new self-stretching technique.

The gain and uniformity measurements of the self-stretched $10 \times 10 \mathrm{~cm}^{2}$ detector are shown in figure 14, and the detector performs just as well as the glued detector. Following these encouraging results we built a $30 \times 30 \mathrm{~cm}^{2}$ detector [3] for further study.

\section{Conclusion and outlook}

In this work we have demonstrated that triple-GEMs are a candidate for instrumenting the high eta part of the forward muon system of CMS. Small prototypes studies in the laboratory and at the beam tests have confirmed expected performance. Large-size prototypes commensurate with the first muon station sizes in CMS have been built and tested in magnetic field. We plan to build the next round of detector prototypes for muon station 2 using simplified construction techniques, which have been validated with small detectors. 
Effective Gain vs Voltage
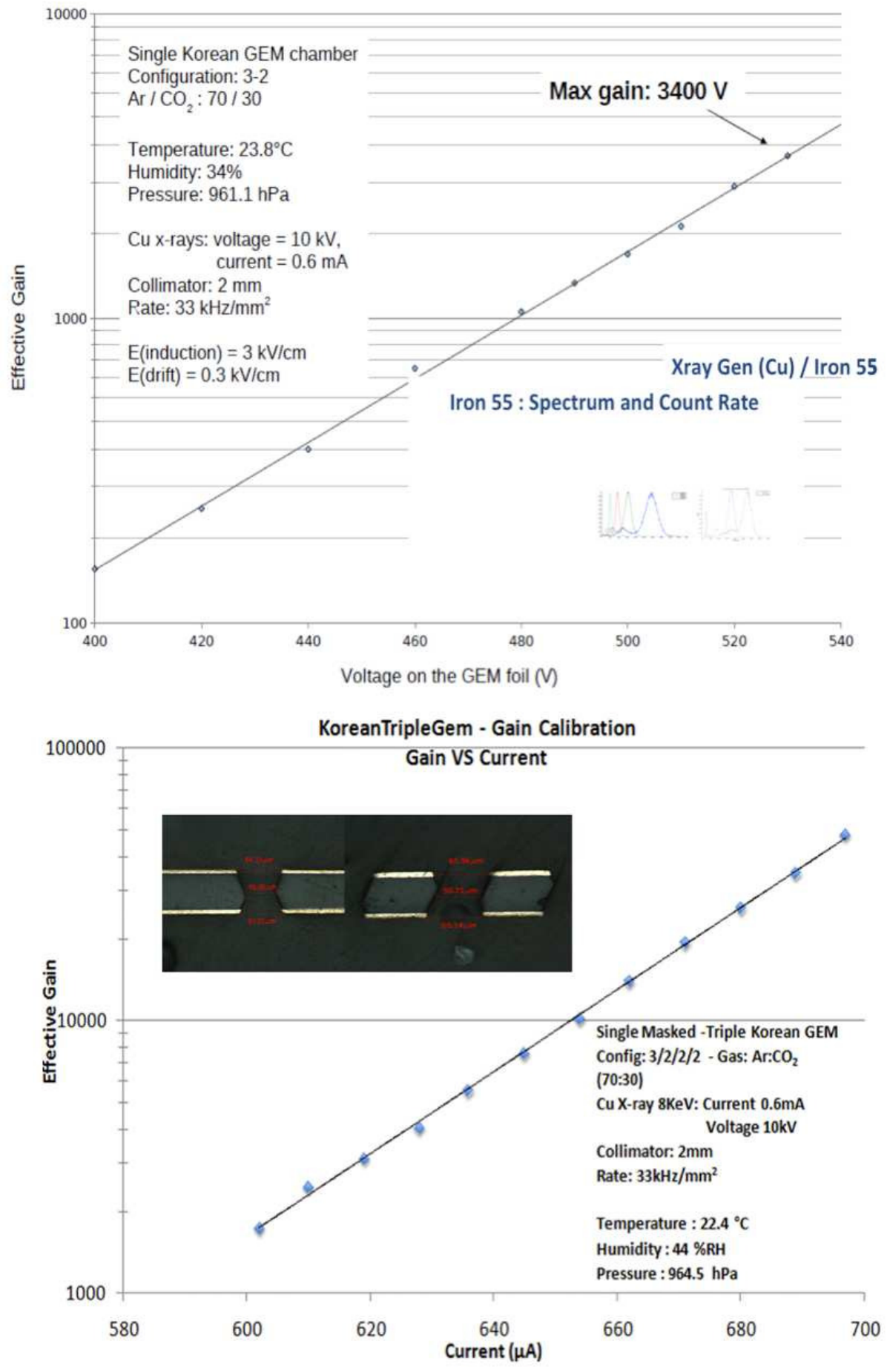

Figure 12. Gain measured on a single GEM foil made in Korea (top) and on a triple GEM using these foils (bottom). 


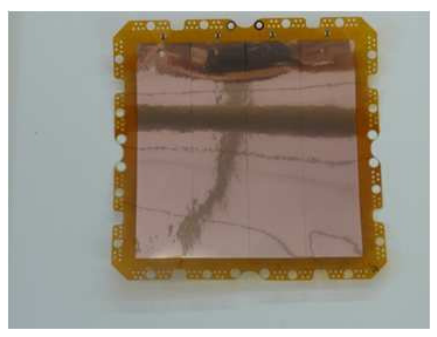

(a)

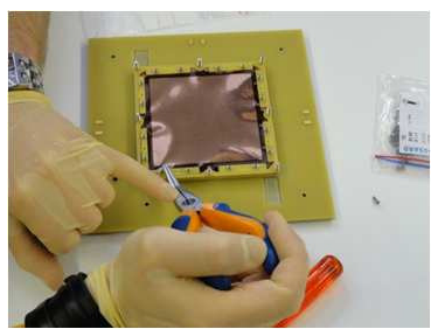

(c)

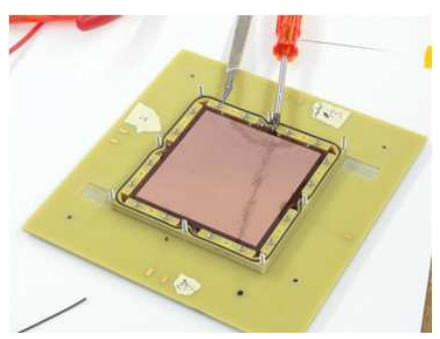

(e)

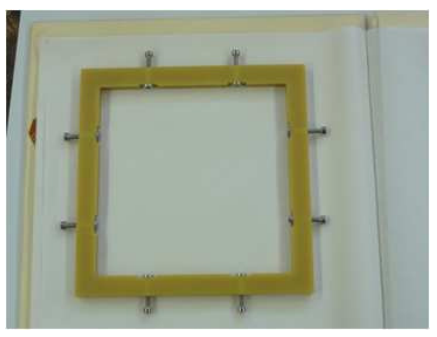

(b)

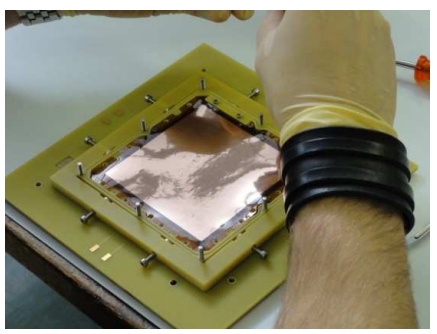

(d)

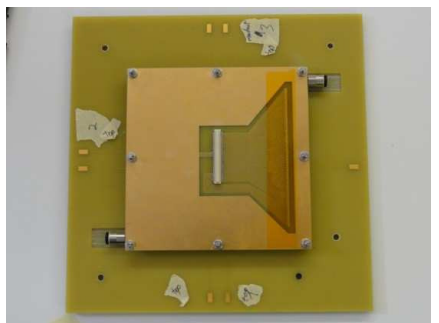

(f)

Figure 13. The self-stretching triple-GEM assembly technique: (a) GEM foil before assembly; (b) external frame for foil stretching; (c) mounting the GEM foils; (d) stretching the foils; (e) stretched GEM; (f) completed detector including readout board.

Gain Calibration ArCO2 (70:30)



Figure 14. Gain and uniformity measurements of the self-stretched prototype. 


\section{Acknowledgments}

This work has partly been performed in the framework of the RD51 Collaboration. Many thanks to Dr. Sijin Qian for a careful reading of manuscript.

\section{References}

[1] M. Tytgat et al., Construction and performance of large-area triple-GEM prototypes for future upgrades of the CMS forward muon system, in Proceedings of the 2011 IEEE Nuclear Science Symposium, NSS-MIC2011 N19-7 RD51-Note-2011-012, Valencia Spain (2011) [arXiv: 1111.7249].

[2] CMS collaboration, S. Chatrchyan et al., The CMS experiment at the CERN LHC, 2008 JINST 3 S08004.

[3] D. Abbaneo et al., Characterization of GEM detectors for application in the CMS muon detection system, in Proceedings of the 2010 IEEE Nuclear Science Symposium, RD51-Note-20112010.5874, Knoxville U.S.A. [arXiv: 1012.3675].

[4] D. Abbaneo et al., Construction of the first full-size GEM-based prototype for the CMS high- $\eta$ muon system, in Proceedings of the 2010 IEEE Nuclear Science Symposium, RD51-Note-2010-0082010.5874, Knoxville U.S.A. [arXiv: 1012 .1524].

[5] D. Abbaneo et al., Construction and performance of full scale GEM prototypes for future upgrades of the CMS forward muon system, in Proceedings of the $13^{\text {th }}$ ICATPP Conference on Astroparticle, Particle, Space Physics and Detectors for Physics Applications, Como Italy October 3-7 2011.

[6] TOTEM collaboration, Total cross-section, elastic scattering and diffraction dissociation at the Large Hadron Collider at CERN: TOTEM technical design report, CERN-LHCC-2004-002, CERN, Geneva Switzerland (2004) [Addendum CERN-LHCC-2004-020].

[7] M. Alfonsi et al., Fast triggering of high-rate charged particles with a triple-GEM detector, Nucl. Instrum. Meth. A 535 (2004) 319.

[8] S.A. Tupputi et al., Performance studies of large-area triple-GEM prototypes for future upgrades of the CMS forward muon system, in Proceedings of EPS-HEP 2011, Grenoble France July 21-27 2011.

[9] CMS collaboration, The muon project, CMS technical design report, CERN-LHCC-97-32, CERN, Geneva Switzerland (1997) [CMS-TDR-003].

[10] I. Furic, Studies for the forward muon trigger algorithm, in CMS Upgrade Workshop, Fermilab, Batavia U.S.A. November 7-10 2011.

[11] F. Sauli, GEM: a new concept for electron amplification in gas detectors, Nucl. Instrum. Meth. A 386 (1997) 531.

[12] P. Aspell et al., VFAT2: a front-end system on chip providing fast trigger information, digitized data storage and formatting for the charge sensitive readout of multi-channel silicon and gas particle detectors, in Proceedings of the Topical Workhop on Electronics for Particle Physic (TWEPP2007), Prague Czech Republic September 3-7 2007.

[13] S. Duarte Pinto et al., Progress on large area GEMs, 2009 JINST 4 P12009 [arXiv: 0909. 5039]; M. Villa et al., Progress on large area GEMs (VCI 2010), Nucl. Instrum. Meth. A 628 (2011) 182 [arXiv: 1007.1131].

[14] New flex technolgy co.ltd. webpage, http://www.newflex.co.kr/, Gyeong Korea. 